\title{
Centro de Atenção Psicossocial de álcool e outras drogas: práticas desenvolvidas pelos profissionais de saúde
}

\author{
Center for Psychosocial Attention for alcohol and other drugs: \\ practices developed by health professionals
}

\author{
Marina Cristina Zotesso', Lais Oliveira Marques'2 , Sônia Maria Alves de Paiva ${ }^{3}$ \\ Autora para correspondência. Universidade de Marília. Marília, São Paulo, Brasil. ORCID: 0000-0003-3180-1575.marina.zotesso@gmail.com \\ ${ }^{2}$ Pontifícia Universidade Católica de Minas Gerais. Poços de Caldas, Minas Gerais, Brasil. ORCID: 0000-0001-5139-698X. lalaoliveira2@hotmail.com \\ ${ }^{3}$ Centro Estadual de Educação Tecnológica Paula Souza, Fundação de Ensino Octávio Bastos. São Paulo, São Paulo, Brasil. \\ ORCID: 0000-0001-7918-3855. soniaenf@hotmail.com
}

RESUMO | O aumento no consumo de álcool entre a população tem acarretado graves problemas familiares, profissionais e sociais, associado a riscos de alta complexidade, o que levou o Ministério da Saúde a adotar estratégias para a redução de danos; entre as quais, os Centros de Atenção Psicossocial em Álcool e Outras Drogas (CAPSad). O presente estudo teve como objetivo identificar a percepção da equipe de saúde do CAPSad sobre o alcoolismo e descrever as ações que dificultam o atendimento. Realizou-se um estudo qualitativo, descritivo e exploratório, por meio de uma entrevista com nove profissionais de saúde. As Questões norteadoras foram divididas em categorias para análise. Os resultados indicam categorias e subcategorias de análise, tais como: Álcool no contexto social, fatores que dificultam a adesão do usuário, necessidade de qualificação da equipe, entre outras. Dessa forma, evidenciou-se a necessidade de capacitação da equipe através de práticas de educação permanente, favorecendo e estimulando os princípios da reabilitação psicossocial.

PALAVRAS-CHAVE: Alcoolismo. Centros de Atenção Psicossocial. Equipe de Saúde.

\begin{abstract}
The increase in alcohol consumption among the population has caused serious family, professional and social problems, associated with high complexity risks, which led the Ministry of Health to adopt strategies for harm reduction; including the Centers for Psychosocial Care in Alcohol and Other Drugs (CAPSad). The present study aimed to identify the CAPSad health team's perception of alcoholism and describe the actions that make it difficult to attend. A qualitative, descriptive and exploratory study was conducted through an interview with nine health professionals. Guiding questions were divided into categories for analysis. The results indicate categories and subcategories of analysis, such as: Alcohol in the social context, factors that hinder the user's adhesion, the need to qualify the team, among others. In this way, the need for training of the team through permanent education practices was evidenced, favoring and stimulating the principles of psychosocial rehabilitation.
\end{abstract}

KEYWORDS: Alcoholism. Psychosocial Attention Centers. Health Team. 


\section{Introdução}

Encontra-se na literatura científica diferentes estudos relacionados à temática álcool e outras drogas, no entanto este tema parece longe de se esgotar, enquanto prática de pesquisa, por inúmeras razões. Estudo desenvolvido em 2016, por exemplo, mostra relação entre o uso de álcool e motivação para suicídio por homens. O álcool é apontado como um hábito estimulado socialmente principalmente aos meninos, já desde o final da infância (Ribeiro et al., 2016).

abuso de álcool e outras drogas por homens, quando atrelado à ideia de uma vida social conturbada, relaciona-se também com a motivação para o suicídio. (Ribeiro et al., 2016). Dados coletados em estudo sobre tentativas de suicídio atendidas em um Hospital localizado no estado de Minas Gerais (Brasil) mostram que entre os anos de 1998 e 2012 ocorreram mais tentativas de suicídio por homens (334 pessoas, $55,6 \%$ ), pessoas com idade entre 20 a 59 anos e por meio de intoxicação exógena (com uso de alguma substância em quantidade tóxica para o organismo) (Costa et al., 2015).

Em casos de tentativa de suicídio o tempo de internação hospitalar pode variar, assim como o custo da assistência de saúde. (Costa et al., 2015). Faz-se relevante tocar nesse assunto porque a falta de ações como a de prevenção em suicídio, além de poder resultar em muitos óbitos, gera também um custo muito elevado para a saúde. O dinheiro gasto com a assistência hospitalar em casos de tentativa de suicídio poderia ser melhor aplicado com ações, por exemplo, de capacitação de profissionais de saúde no que se refere à prevenção do suicídio, tendo por base o perfil das pessoas que usualmente se sentem motivadas para a tentativa de suicídio, como é o caso dos homens usuários de substâncias psicoativas (Ribeiro et al., 2016).

No que se refere ao uso de substâncias psicoativas por adolescentes verifica-se que o risco para diagnóstico de transtorno do desenvolvimento psicológico é 4,27 vezes maior para aqueles que abusam de substâncias como cocaína/crack, podendo também ocorrer em menor grau nos adolescentes que fazem uso de estimulantes (Rocha et al., 2015).
De forma geral pode-se afirmar que o uso de substâncias psicoativas pode causar diferentes impactos na vida e na saúde das pessoas, sendo necessária a promoção de assistência de saúde qualificada. Visando acolher e prestar um atendimento adequado a essa população foram criados e implantados, no Brasil, os Centros de Atenção Psicossocial (CAPSad), que se constituem em uma das principais estratégias de enfrentamento dos problemas relacionados ao abuso de álcool e outras drogas pela população (Ministério da Saúde, 2004).

O Centro de Atenção Psicossocial em Álcool e Outras Drogas (CAPSad) consiste de uma modalidade de serviço de caráter aberto e comunitário, dotado de equipe multiprofissional e transdisciplinar para atendimento a usuários de substâncias psicoativas e conforme descrito na Portaria $n^{\circ} 3.088$, de 23 de dezembro de 2011 , do Ministério da Saúde, atende pessoas de todas as faixas etárias que apresentem intenso sofrimento psíquico decorrente do uso de crack, álcool e outras drogas. Indicado para municípios ou regiões de saúde com população acima de setenta mil habitantes (Ministério da Saúde, 2011).

O perfil dos usuários do CAPSad costuma ser de maioria do sexo masculino, raça parda, solteiros, com menos de 10 anos de estudo, desempregados ou sem emprego formal e usuários de substâncias como álcool, crack, maconha, cocaína, tabaco ou outras (Matos et al., 2015; Fernandes et al., 2015; Dias et al., 2015; Barbosa et al., 2015; Gonçalves et al., 2017).

O CAPSad deve ofertar uma atenção contínua às pessoas com necessidades relacionadas ao consumo de álcool, crack e outras drogas; ser um lugar de referência de cuidado e proteção para os usuários e familiares em situações de crise e de maior gravidade e produzir, em conjunto com o usuário e seus familiares, um Projeto Terapêutico Singular (PTS), que o acompanhe nos contextos cotidianos, promovendo e ampliando as possibilidades de vida e contar com o serviço de uma equipe, onde cada usuário tenha a seu dispor um profissional de referência, que 0 acolha, sob a ótica da interdisciplinaridade (Ministério da Saúde, 2012). 
A equipe de saúde desse serviço, em princípio, deve promover a (re)inserção social, proteção e suporte de grupo para os usuários do serviço, no processo de reabilitação psicossocial e adequar a oferta dos serviços de acordo com suas necessidades de horários de acolhimento, incluindo os familiares. (Ministério da Saúde, 2012). Sendo assim, o propósito das ações de saúde desenvolvidas no CAPSad é a melhora da situação geral de saúde dos usuários do serviço, além de melhora da vida dessa pessoa, com valorização de sua subjetividade, fortalecendo suas habilidades e potencialidades (Rocha et al., 2015).

Espera-se que as ações desenvolvidas pelos profissionais do CAPSad também estejam voltadas para a estratégia de Redução de Danos, pelo manejo e cuidado diante de comorbidades psiquiátricas ou clínicas das pessoas, com realização de ações de apoio matricial na Atenção Básica, compartilhando a responsabilidade com os profissionais da Rede de Atenção à Saúde (Ministério da Saúde, 2012).

Estudos têm abordado a inserção e as práticas da equipe de saúde, e dos papeis executados pelos profissionais, como o enfermeiro e o psicólogo âmbito dos serviços substitutivos de saúde mental, evidenciando que, para sua atuação tem sido exigido um novo saber, construído a partir da prática interdisciplinar, o que tem contribuído para torná-lo um profissional mais autônomo, porém requer qualificação, uma vez que, se antes suas funções eram precisas e bem definidas, a inserção de novos modelos de atendimento, exige dele, responsabilidades inexploradas e pouco precisas (Vargas, Oliveira \& Duarte, 2011); (Vargas, Bittencourt, Rocha \& Oliveira, 2013).

Esse processo de reinserção social demanda do profissional uma prática compreensiva, reflexiva e criativa, onde a escuta e o diálogo passam a ser peças fundamentais no tratamento e na construção do vínculo profissional-usuário (Soares, Villela, Borba, Brusamarello \& Maftum, 2011 ).

Ainda segundo os autores, a proposta de trabalho no CAPS inclui, além da pessoa com transtorno mental, a família e a sociedade, exigindo atividades direcionadas a um grupo ampliado, para o qual os profissionais deverão utilizar o saber acumulado na profissão e agregá-lo ao que for necessário na prática cotidiana do CAPSad.

Desse modo o presente estudo teve como objetivo, identificar a percepção da equipe de um CAPSad sobre o alcoolismo, descrever as ações de saúde implementadas pela equipe e as dificuldades no atendimento.

\section{Método}

Trata-se de estudo qualitativo descritivo e exploratório atrelado a uma pesquisa matricial financiada pelo Fundo de Incentivo à Pesquisa (FIP) e desenvolvida no CAPSad de um município no interior de Minas Gerais.

O estudo de caso utilizou da metodológica baseada em Minayo (2013). A amostra compôs-se de 9 profissionais de saúde: 1 enfermeiro, 2 assistentes sociais, 4 psicólogos, 1 psiquiatra e 1 terapeuta ocupacional, entrevistados face-a-face após assinatura do Termo de Consentimento Livre e Esclarecido (TCLE). Para realização das entrevistas tomou-se por base as questões norteadoras do estudo.

Como critérios de inclusão foram considerados os profissionais com vínculo empregatício com o CAPSad no qual a pesquisa foi desenvolvida e, como critérios de exclusão, a ausência no dia de coleta dos funcionários afastados por motivos de saúde e/ou férias. Realizou-se um pré-teste para a verificação da confiabilidade e pertinência do instrumento de coleta de dados elaborado pelas pesquisadoras, bem como para averiguação de possíveis adequados no instrumento para melhor alcance dos objetivos da pesquisa.

As questões norteadoras do estudo foram: 1) Qual a sua visão sobre o alcoolismo? 2) Quais são as suas atividades aqui no CAPSad? 3) Que dificuldades você enfrenta no atendimento aos usuários do CAPSad?

As entrevistas foram realizadas em local reservado para assegurar a privacidade e $\circ$ anonimato das informações dos participantes. As mesmas foram gravadas somente após consentimento dos 
participantes, e teve como tempo médio de coleta de dados de aproximadamente 50min. As gravações foram posteriormente transcritas pelas pesquisadoras para análise dos resultados.

Na análise dos dados fez-se leitura exaustiva das transcrições. Posteriormente elaborou-se uma planiIha com essas transcrições para extração dos núcleos de sentido e elaboração das categorias de análise. As categorias de análise foram elaboradas conforme referencial teórico de Minayo (2013). As categorias foram selecionadas mediante as falas similares dos entrevistados, sendo acopladas em grupos e utilizando-se da análise do conteúdo.

Os riscos atribuídos à participação neste estudo referiram-se à possibilidade de constrangimento dos profissionais em falar sobre questões pertinentes à prática profissional dentro do próprio ambiente de trabalho e a possibilidade de que entrevistados fossem identificados por outros profissionais do estudo. Os benefícios em participar da pesquisa incluíram a colaboração para o avanço da ciência e teorização de questões que permeiam a prática profissional no serviço de álcool e outras drogas.

A estratégia dos pesquisadores para minimizar os riscos partiu da solicitação de um local reservado no serviço de saúde para a realização das entrevistas, sem que outros profissionais pudessem acessar - local da coleta de dados, ou ouvir o que cada entrevistado dizia em sua entrevista.

Como todo estudo qualitativo a limitação do presente estudo está no fato de ter sido utilizada amostra pequena, porém considera-se que a contribuição dos participantes foi de grande valia para a pesquisa e conhecimento das ações da equipe de saúde mental no CAPSad.

\section{Resultados}

As categorias de análise elaboradas foram: Categoria 1 - Álcool no contexto social, Categoria 2 - As práticas dos profissionais no contexto dos CAPSad e Categoria 3 - Fatores que dificultam a adesão do usuário nos CAPSad.

\section{Categoria 1 - Álcool no contexto social}

Dessa categoria emergiu duas subcategorias: a) o alcoolismo como um problema social e b) ambiente familiar; conforme abaixo:

a) $O$ alcoolismo no contexto da sociedade

O uso abusivo de álcool está ligado a diversas consequências para $\circ$ indivíduo que o consome para aqueles que estão à sua volta e para a sociedade como um todo, acarretando problemas familiares, no trabalho, violência, acidentes e como droga lícita é aceita socialmente, abrindo portas para as drogas ilícitas. $O$ alcoolismo, portanto, traduz-se em um conjunto de problemas relacionados com o consumo excessivo e prolongado do álcool e todas as consequências daí decorrentes (Oliveira, 2009).

\section{E 1: "O álcool pra mim é a droga, que abre as portas} pras demais drogas na sociedade."

E2: "Um problema que atinge a maioria da sociedade, grande parte da população."

Ainda segundo o autor, o álcool é uma droga subestimada, pois a sociedade encara-o como parte de uma vida "normal" dos indivíduos, incluindo-o praticamente em todos os ambientes e situações. A criança cresce aprendendo que beber uma cerveja nos encontros sociais e familiares é um ato social normal e na adolescência, esse comportamento se iniciará como um marco que indica a entrada na vida adulta.

\section{b) Ambiente familiar}

A literatura faz referências ao alcoolismo parental como um importante fator de risco para o desenvolvimento de problemas relacionados à bebida alcoólica na adolescência, divórcio dos pais, estrutura e relação afetiva familiar, dificuldade de comunicação com os pais na adolescência, violência intra-familiar; famílias que vivenciam fortes pressões socioeconômicas e as de padrões educacionais rígidos e punitivos com os filhos. (Nunõ-Guitièrrez \& Gonzalez-Fortaleza, 2004; Horta, Horta \& Pinheiro, 2006; Wandekoken, Vicente \& Siqueira, 2011 ). 
E4 “... está vinculado a uma desestrutura familiar, muitos casos que a gente pega aqui você percebe que já tem uma desestrutura familiar envolvida.

\section{Categoria 2 - As práticas dos profissionais no contexto dos CAPSad}

A segunda categoria evidenciou as práticas dos profissionais no contexto de CAPSad, atividades essas enfatizando o processo de reabilitação; destacando-se o acolhimento, os atendimentos individuais e grupais, o atendimento familiar, oficinas terapêuticas, orientação sobre direitos e sobre os problemas relativos ao álcool, tratamento medicamentoso, visitas domiciliares e busca ativa.

E 1: "Nós fazemos desde uma intervenção individual, onde você escuta para saber qual é a queixa, ou quais as situações vulneráveis das recaídas ou por que houve as recaídas, a questão emocional.Outro momento também é o trabalho em grupo, onde se faz uma terapia de grupo, onde também você está escutando e o grupo está se colocando, sobre a doença sobre as suas condições, sobre as suas limitações, sobre a situação vulnerável que ele se encontra, e todas as situações de risco que ele se encontra.

Fazemos trabalhos em oficinas terapêuticas, que é uma oportunidade das pessoas que você proporciona de estarem nessas oficinas pensando no seu tratamento, a oficina tem essa finalidade, de você poder estar organizando a sua vida."

E 3: "Aqui a gente faz o acolhimento, coordenação nas oficinas, eu faço visita domiciliar, busca ativa, então quer dizer, quando a pessoa não vem, a gente vai atrás, tenta mobilizar, conscientizar para o tratamento.

E 6: "Eu oriento sobre os sintomas da dependência, da abstinência, oriento sobre os cuidados mesmo com a saúde, o sintoma da dependência e da abstinência". Trabalha-se também com a ansiedade, com o controle sobre a doença, e com a família,... não adianta tratar

só o paciente, tem que cuidar da família também."

Essas respostas corresponderam às atividades pautadas nas ações programáticas da estratégia do Ministério da Saúde e são tidas como importantes para $\circ$ processo de reabilitação psicossocial e de reintegração do usuário na comunidade, exigindo um novo paradigma, baseado nas necessidades e demandas do usuário do CAPSad e na interdisciplinaridade, integrado a um trabalho em rede.

Segundo Leal e Antoni (2013), a participação dos familiares do usuário no CAPSad é fundamental para a sua compreensão sobre a doença e diminuição da sobrecarga do convívio com a condição mental e social do membro adoecido, enquanto a busca ativa deve ser uma prioridade do CAPSad, considerando a baixa assiduidade e $\circ$ alto índice de abandono característicos dessa população, ao passo que a visita domiciliar por sua vez possibilita uma aproximação da equipe com a realidade social do usuário.

\section{Categoria 3 - Fatores que dificultam a adesão do usuário nos CAPSad}

A terceira categoria referiu-se às dificuldades da adesão do usuário no serviço segundo a equipe, da qual originou as subcategorias: a) a não percepção da doença; b) o uso da droga como busca de alívio para o sofrimento e pela falta de um projeto de vida; c) licitude da droga e d) necessidade de qualificação na área.

\section{a) A não percepção da doença}

As falas da equipe se referiram à dificuldade do usuário em perceber os efeitos do consumo do álcool e o impacto do uso sobre seu padrão de vida pessoal e familiar, o que justifica a sua não adesão ao tratamento. É consenso na literatura, o baixo índice de adesão pelos dependentes químicos, sendo que muitos iniciam o tratamento, mas poucos 0 mantêm. Um dos fatores se deve ao fato do início cada vez mais precoce do consumo do álcool, em convívio com pessoas, por vezes do próprio ambiente familiar que bebiam; como já mencionado (LimaRodríguez, Guerra Martín, Domínguez Sánchez \& Lima Serrano, 2015).

Segundo os autores, nesta primeira etapa, pré-contemplativa, os futuros alcoólatras consideravam-se bebedores sociais, o que os impedia de perceber ou interpretar seu modelo de consumo de álcool como sinal ou sintoma de doença e uma vez perdida a capacidade de controlar o consumo, começa a negação. $O$ processo de aceitação inicia-se quando a pessoa começa a perceber que tem uma doença. 
A continuidade do tratamento caracteriza-se como um percurso difícil de atingir, frente à presença de obstáculos e da recaída, pois as dificuldades em abandonar a bebida falam mais alto que o desejo de não beber. Para vencer estes obstáculos é necessário antes de tudo que o dependente se conscientize de sua doença e dos males que esta causa na vida profissional, nos relacionamentos e no convívio familiar (Monteiro, Fé, Moreira, Albuquerque, Silva \& Passamani, 2011).

Para Souza, Kantorski e Mielke (2006) a adesão dos usuários inseridos no CAPSad ao tratamento, está diretamente relacionada à capacidade de se construir um bom vínculo entre o usuário e o profissional de saúde.

E 1: “... a recuperação está na faixa de uns $30 \%$ para baixo". "... e o álcool passa a ser um alívio, ele cria um personagem no momento em que usa, e tem seus benefícios diante do uso dessa droga, mas ao mesmo tempo ele entra em contradição com os prejuízos que - álcool vai arrastando, tanto prejuízos psíquicos como clínicos da sua própria saúde."

b) O uso da droga como busca de alívio para o sofrimento e pela falta de um projeto de vida

De acordo com a equipe, os usuários buscam o álcool como uma forma de lidar com o sofrimento psíquico; com as dificuldades de operar planos e definir um sentido de vida, daí a importância das equipes se atentarem para as demandas dos usuários, através do acolhimento, do vínculo, da escuta, para conhecer melhor a realidade, a história de vida, e identificar as fragilidades desses indivíduos, como estratégia para auxiliá-los no alívio do sofrimento (Cardoso, Taccolini, Tansini, Vieira \& Hirdes, 2014).

E 5: "É, primeiro a quebra de rotina que eles tem né. Que é a busca do alivio pelo álcool, a dificuldade de dar significação à vida."

c) Licitude da droga

O controle do consumo de álcool como substância lícita, vai depender da motivação do usuário. Esse desejo representa a premissa básica para que o tratamento tenha sucesso e segundo Pinho (2009) depende muito do compartilhamento de responsabilidades do usuário e equipe, fundamentado no trabalho interdisciplinar.

E 8: "Acho que o maior desafio é a licitude da droga, no caso do álcool que estamos falando, porque é aceito socialmente."

A falta de iniciativa e/ou o engajamento do paciente é uma limitação que impede a continuidade no tratamento; no entanto, se o paciente tiver visibilidade total de sua condição, bem como da resolução do mesmo e comprometido com o suporte para redução de dados que visam diminuir o consumo de álcool, maior será a resolutibilidade de sua doença (Monteiro, Fé, Moreira, Albuquerque, Silva, \& Passamani, 2011).

Os pacientes que iniciam o tratamento apenas em decorrência de influências externas, como pressão familiar e de amigos, comorbidades clínicas e ordens judiciais, possuem maior dificuldades em aderir ao tratamento, pois não se sentem realmente motivados e comprometidos para o mesmo (Brasil, 2003).

Portanto, cabe à equipe não ter como meta a abstinência como único objetivo a ser alcançado, mas privilegiar a redução de danos ao reconhecer cada usuário em suas singularidades e traçar estratégias que $o$ ajudem a perceber outras formas de viver $e$ as consequências do uso abusivo do álcool em seu cotidiano (Brasil, 2003).

\section{d) Necessidade de qualificação da equipe}

A carência de preparo formal dos profissionais, entre eles, os enfermeiros para o cuidado de dependentes de substâncias psicoativas deve-se, ao fato de muitos cursos de graduação não oferecerem conteúdos teóricos atualizados que acompanhem os avanços dessa área de conhecimento e, portanto, não preparam adequadamente os profissionais, - que os leva a reproduzir a lógica reducionista (Vargas, Oliveira \& Duarte, 2011).

E 1: “... é um desafio, a gente aprende todos os dias e ao mesmo tempo a gente troca experiências entre os profissionais que trabalham, escuta, e o próprio usuário ensina muito." 
E 2: "Então eu sempre procuro ler, estou querendo agora fazer uma especialização justamente para ficar um pouco mais preparada pra estar aqui."

E 3: “... especificamente dependência química pra mim era uma coisa nova, e eu tenho aprendido muito."

E 5: "Olha a gente nunca esta, eu nunca me sinto preparada, a gente esta sempre se preparando."

E 9: "Eu me consideraria preparada talvez se eu me especializasse na área. Porque aparentemente é uma área assim simples, mas é quando você esta no dia a dia você vê que envolve muitas outras coisas, envolver conhecimento."

Destaca-se a necessidade de se criar estratégias e dispositivos de gestão, atenção e formação em saúde mental, que possam impactar na forma dos serviços se articularem em rede. Uma dessas possibilidades é o apoio matricial para as equipes das Estratégias de Saúde da Família (ESFs), por meio da integração ensino-serviço dos Programas de Residências Multiprofissionais, no campo da saúde mental, com as equipes de atenção básica e cursos de especialização na área (Cortez et al., 2014).

A insuficiente qualificação para $\circ$ trabalho limita a atuação da equipe, conforme os pressupostos e diretrizes da atual Política sobre Drogas. Isso resulta em dificuldades no planejamento do cuidado e interfere negativamente na relação terapêutica.

\section{Discussão dos resultados}

No modelo psicossocial, o sujeito é tido como um agente ativo, portanto, é fundamental que se estabeleça com o usuário, uma relação, respeitando-se a sua subjetividade, historicidade e suas vivências, de acordo com os objetivos da reabilitação psicossocial, na prevenção do estigma, discriminação e reestruturação de suas potencialidades, de modo a auxiliá-lo a estar apto para o exercício de seus direitos e cidadania (Pinho, 2009).

A inclusão dos familiares no plano terapêutico e compreensão da doença são essenciais no enfrentamento das dificuldades como conseqüência do uso de álcool pelo paciente e, como forma de apoiá-lo na superação do problema e em sua recuperação.

Em relação às práticas assistenciais, constatou-se que as falas se mantiveram alinhadas à proposta da reabilitação psicossocial preconizada pelas diretrizes do Ministério da Saúde (Brasil, 2004). A equipe de saúde utilizou como estratégia de incentivo à adesão ao serviço o desenvolvimento de oficinas de caráter terapêutico, de entretenimento e de aprendizagem de ofícios, visando à profissionalização, conforme aponta Pinho (2009). No entanto, os relatos demonstram, ainda, um tipo individualizado de intervenção em contraposição ao tipo interdisciplinar, que é considerado como exigência na atenção psicossocial (Silva, 2014).

A capacidade prática do enfermeiro relativa às habilidades necessárias às intervenções psicossociais não ficaram evidenciadas. De acordo com as falas, os profissionais da enfermagem se restringem às especificidades técnicas do cuidado ao usuário do CAPSad.

Em relação ao tema, Vargas, Oliveira e Duarte (2011) já apontavam possíveis causas para esse despreparo por parte dos profissionais da enfermagem. Segundo os autores, a pouca exploração dessas habilidades durante o período de formação gera deficiências no conhecimento e, consequentemente, reduz a capacidade de atuação profissional ao atendimento de caráter clínico e administrativo, o que pouco contribui para a adesão do usuário ao CAPSad.

\section{Limites e perspectivas do estudo}

No presente estudo não foi possível analisar o material coletado à luz da abordagem da redução de danos, bem como na perspectiva sóciooantropológica para uso de álcool. Nesse sentido avaliou-se que novas pesquisas são necessárias para ampliar o enfoque das práxis entre a Enfermagem e Psicologia. Dessa forma possibilitará aprofundar também os estudos socioantropológicos e propiciar novas interfaces epistemológicas, fundamentando as propostas de trabalho nos campos da enfermagem e da psicologia. 


\section{Considerações finais}

As atividades do projeto terapêutico realizadas pela equipe do CAPSad devem ser interdisciplinares e caracterizarem-se por ações fundamentadas na reabilitação psicossocial. No entanto, atuar no CAPSad requer muitos conhecimentos e habilidades que a literatura científica ainda não se apropriou completamente.

Os objetivos deste estudo foram alcançados e evidenciam a necessidade de capacitação da equipe profissional para atender as demandas dos usuários no serviço. Atualmente, com a expansão dos cursos de graduação no país, é possível pensar parcerias entre os municípios e as universidades mais próximas, favorecendo a oferta de cursos de especialização em diferentes áreas do conhecimento, além de outras formas de capacitação.

Para tanto, sugere-se que os gestores municipais e os dos CAPSad promovam estratégias de capacitação dos profissionais que atuam nos serviços, com incentivos e favorecimento à participação da equipe nas ações promovidas em parceria. Essas ações atenderiam o proposto pela Portaria GM/MS $n^{\circ}$ 336 , que determina que os profissionais que compõem a equipe de saúde mental devem possuir especialização na área.

Cabe também aos profissionais o compromisso e a responsabilização pela sua formação e a busca contínua de aprimoramento profissional, que lhes possibilite oportunidades de intervenções que atendam com segurança às necessidades dos usuários e familiares, alinhados à prática do paradigma da reforma psiquiátrica.

\section{Contribuições das autoras}

Zotesso, M. C. participou da concepção, coleta de dados, interpretação dos resultados, delineamento, busca e análise estatística dos dados da pesquisa, interpretação dos resultados e redação do artigo científico. Marques, L. O. participou da coleta e interpretação dos dados. Paiva, S. M. A. participou da interpretação dos resultados, análise estatística dos dados da pesquisa e redação do artigo científico.

\section{Conflitos de interesses}

Nenhum conflito financeiro, legal ou político envolvendo terceiros (governo, empresas e fundações privadas, etc.) foi declarado para nenhum aspecto do trabalho submetido (incluindo mas não limitandose a subvenções e financiamentos, participação em conselho consultivo, desenho de estudo, preparação de manuscrito, análise estatística, etc.).

\section{Referências}

Barbosa, G. C., Oliveira, M. A. F., Moreno, V., Padovani, C. R., Claro, H. G., \& Pinho, P. H. (2015). Satisfação de usuários em um Centro de Atenção Psicossocial em álcool e outras drogas. Revista Portuguesa de Enfermagem de Saúde Mental (14), 31 -37. doi: $10.19131 /$ rpesm.0103

Cardoso, M. P., Agnol, D. R., Taccolini, C., Tansini, K., Vieira, A., \& Hirdes, A. (2014). A percepção dos usuários sobre a abordagem de álcool e outras drogas na atenção primária à saúde. Aletheia, (45), 72-86.

Cortez, F. C., Terra, M. G., Pires, F. B., Heinrichi, J., Machado, K. L., Weiler, T. H. \& Padoin, S. M. M. (2014). Atenção a usuários de álcool e outras drogas e os limites da composição de redes. Revista Eletrônica de Enfermagem 16(1), 84-92. doi: 10.5216/ree.v16i1.20279

D'Ramos, A. O. S. (2014). Assistência de enfermagem na atenção psicossocial. Especialista (Trabalho de Conclusão de Curso). Departamento de Enfermagem da Universidade Federal de Santa Catarina, Florianópolis, SC, Brasil.

Da Costa, S. P., Chavaglia, S. R. R., Amaral, E. M. S. \& Silveira, R. E. (2015). Internações e Gastos relacionados ao Suicídio em um hospital público de ensino. Revista de Enfermagem e Atenção à Saúde, 4(2), 20-32.

De Matos, F. V., Silva, L. M. P., Silveira, A. R., \& Sampaio, C. A. (2015). Perfil dos usuários do centro de atenção psicossocial álcool e outras drogas de Montes Claros, Minas Gerais, Brasil. Revista Unimontes Científica, $17(1), 28-38$.

Dias, M. T. G., Meneghel, S. N., Ceccon, R. F., Junges, E. M. G., Ávila, M. Y. B., Rosa, S., ... Canto, R. B. B. (2015). Usuários de um centro de atenção psicossocial álcool e drogas, Porto Alegre, RS: perfil e desfecho. In A. A. Ferla, C. M. F. Rocha, M. T. G. Dias \& L. M. Santos (Orgs.), Redes vivas de educação e saúde: relatos e vivências da integração universidade e sistema de saúde ( 1 a ed., pp. 156-165). Porto Alegre: Rede Unida. 
Fernandes, P. A. J., Gomes, E. S., Lima, M. V. P., Silva, M. L., Barbosa, V. M., \& Pachú, C. O. (2014). Caps ad: drogas psicoativas promotoras de dependência entre assistidos. Revista de Biologia \& Farmácia e Manejo Agrícola, 10(3), 31-34.

Horta, R. L., Horta, B. L., \& Pinheiro, R. T. (2006). Drogas: Famílias que protegem e que expõem adolescentes ao risco. Jornal Brasileiro de Psiquiatria, 55(4), 268-272. doi: $10.1590 /$ S0047-20852006000400002

Lima-Rodríguez, J. S., Guerra-Martín, M. D., DomínguezSánchez, I., \& Lima-Serrano, M. (2015). Resposta da pessoa doente alcoólatra frente à sua doença: perspectivas de pacientes e familiares. Revista LatinoAmericana de Enfermagem, 23(6), $1165-72$. doi: $110.1590 / 0104-1169.0516 .2662$

Minayo, M. C. S. (2013). O Desafio do Conhecimento (12a ed.). São Paulo: Hucitec.

Ministério da Saúde. (2011). Portaria $n^{\circ} 3.088$, de 23 de dezembro de 2011 , republicada em 21 de maio de 2013: dispõe sobre os Centros de Atenção Psicossocial e os organiza em diferentes modalidades. Recuperado de http://www.bvsms.saude.gov.br/bvs/ saudelegis/gm/2011/prt3088_23_12_2011_rep. html

Ministério da Saúde (2012). Portaria $n^{\circ} 130$, de 26 de janeiro de 2012, redefine o Centro de Atenção Psicossocial de Álcool e outras Drogas $24 \mathrm{~h}$ (CAPS AD III) e os respectivos incentivos financeiros. Recuperado de http://www.bvsms.saude.gov.br/bvs/saudelegis/ gm/2012/prt0130_26_01_2012.html

Ministério da Saúde; Secretaria de Atenção à Saúde; Departamento de Ações Programáticas Estratégicas. (2004). Saúde mental no SUS: os centros de atenção psicossocial. Brasília: Ministério da Saúde.

Ministério da Saúde; Secretaria Executiva; Coordenação Nacional de DST e AIDS. (2003). A Política do Ministério da Saúde para atenção integral a usuários de álcool e outras drogas. Brasília: Ministério da Saúde

Monteiro, C. F. S., Fé, L. C. M., Moreira, M. A. C., Albuquerque, I. E.M., Silva, M. G., \& Passamani, M. C. (2011). Perfil sociodemográfico e adesão ao tratamento de dependentes de álcool em CAPS-ad do Piauí. Escola Anna Nery, 15(1), 90-95.

Nunõ-Guitièrrez, B. L., \& Gonzalez-Fortaleza, C. (2004). La representación social que orienta las decisiones paternas al afrontar el cosnumo de drogas de sus hijos. Salud Pública de México, 46(2), 123-131.
Oliveira, B. P. (2009). Alcoolismo: vivência familiar de uma doença social (Dissertação de mestrado). Faculdade de Letras da Universidade do Porto, Porto, Portugal.

Oliveira, G. C., Nasi, C., Lacchini, A. B., \& Schneider, J. F. (2015). A reabilitação psicossocial: processo de reconstrução da subjetividade do usuário de drogas. Revista Enfermagem Ueri, 23(6), 811-816.

Pinho, P. H. (2009). Os desafios na atenção dos usuários de álcool e outras drogas e a reabilitação psicossocial (Dissertação de mestrado). Escola de Enfermagem da Universidade de São Paulo, São Paulo, SP, Brasil.

Ribeiro, D. B., Terra, M. G., Schineider, J. F., Soccol, L. S., Camillo, L. A., \& Plein, F. A. D. S. (2016). Motivos da tentativa de suicídio expressos por homens usuários de álcool e outras drogas. Revista Gaúcha de Enfermagem, $37(1), 1-7$.

Rocha, F. V., Oliveira, R. L., Brum, D. A. S., Cavalcante, R. B., \& Machado, R. M. (2015). Epidemiologia dos transtornos do desenvolvimento psicológico em adolescentes: uso de álcool e outras drogas. Revista Rene, 16(1), 54-63.

Soares, R. D., Villela, J. C., Borba, L. O., Brusamarello, T., \& Maftum, M. A. (2011). O papel da equipe de enfermagem no centro de atenção psicossocial. Escola Anna Nery, 15(1), 110-115.

Souza, J., Kantorski, L.P., \& Mielke, F. B. (2006). Vínculos e redes sociais de indivíduos dependentes de substâncias psicoativas sob tratamento em CAPS AD. SMAD. Revista eletrônica saúde mental álcool e drogas, 2(1).

Vargas, D., Bittencourt, M. N., Rocha, F. M., \& Oliveira, M. A. F. (2013). Representação social de enfermeiros de centros de atenção psicossocial em álcool e drogas (CAPS AD) sobre o dependente químico. Escola Anna Nery, 17(2), 242-248. doi: 10.1590/S1414$\underline{81452013000200006}$

Vargas, D., Oliveira, M. A. F., \& Duarte, F. A. B. (2011). A inserção e as práticas do enfermeiro no contexto dos Centros de Atenção Psicossocial em Álcool e Drogas (CAPS AD) da cidade de São Paulo, Brasil. Revista Latino-Americana de Enfermagem, 19(1), 115-122.

Wandekoken, K.D., Vicente, C. R., \& Siqueira, M. (2011). Alcoolismo parental e fatores de risco associados. SMAD. Revista eletrônica saúde mental álcool e drogas, 7(3), $161-167$.

Wheeler, D. P. \& Bragin, M. (2007). Bringing it all back home: Social work and the challenge of returning veterans. Health and Social Work, 32(4), 297-300. 